\title{
MicroRNA-495 confers inhibitory effects on cancer stem cells in oral squamous cell carcinoma through the HOXC6-mediated TGF- $\beta$ signaling pathway
}

\author{
Xiaolong You', Zhengyu Zhou ${ }^{2}$, Wen Chen ${ }^{3}$, Xiaoyong Wei ${ }^{4}$, Heqiang Zhou ${ }^{1}$ and Wenzheng Luo ${ }^{1 *}$
}

\begin{abstract}
Background: Oral squamous cell carcinoma (OSCC) is associated with high morbidity and ranks sixth among malignancies worldwide. Increasing evidence suggests that microRNAs (miRNAs or miRs) play a critical role in regulating cancer stem cells (CSCs), which drive the proliferation and spread of OSCC. Therefore, based on the alteration of aberrantly expressed miR-495 and homeobox C6 (HOXC6) by Gene Expression Omnibus (GEO) analysis, we subsequently explore the potential effect of miR-495 on the progression of CSCs in OSCC.

Methods: After the isolation of CSCs from the clinical tissue samples of OSCC patients, the expression of miR-495 and HOXC6 was determined, followed by the validation of the relationship between miR-495 and HOXC6. Subsequently, gain- and loss-function approach was performed to detect the role of miR-495 and HOXC6 in cell proliferation, migration, invasion, cell cycle entry, apoptosis, and epithelial-mesenchymal transition (EMT) of CSCs in OSCC, as well as the tumor growth in vivo.

Results: HOXC6 was highly expressed while miR-495 was poorly expressed in OSCC. HOXC6 was verified to be a target gene of miR-495, and miR-495 could inhibit the activation of the TGF- $\beta$ signaling pathway. CSCs with miR495 overexpression or HOXC6 silencing exhibited reversed EMT process; reduced abilities of proliferation, migration, and invasion; and promoted cell apoptosis in vitro. Moreover, inhibited tumor growth was observed in vivo after injection with miR-495 agomir or sh-HOXC6. In contrast, the downregulation of miR-495 showed an induced role in the progression of OSCC.
\end{abstract}

Conclusion: These findings suggest that miR-495 may suppress HOXC6 to inhibit EMT, proliferation, migration, and invasion while promoting apoptosis of CSCS in OSCC by inhibiting the TGF- $\beta$ signaling pathway.

Keywords: microRNA-495, Oral squamous cell carcinoma, Cancer stem cells, Homeobox C6, TGF- $\beta$ signaling pathway, Migration, Invasion, Epithelial-mesenchymal transition

\section{Background}

Oral squamous cell carcinoma (OSCC) is one of the most prevalent types of malignant tumor in the oral system. The 5-year survival rate of patients with OSCC is as low as $60 \%$ and has scarcely improved over the last 15 years [1]. The likelihood of recurrent OSCC is

\footnotetext{
* Correspondence: Iwzluowenzheng@126.com

${ }^{1}$ Department of Head and Neck Surgery, Jiangxi Cancer Hospital, No. 519, Beijing East Road, Nanchang 330029, Jiangxi Province, People's Republic of China

Full list of author information is available at the end of the article
}

associated with several predictive factors including tumor stage, depth of tumor penetration, positive surgical margins, extracapsular spread, and perineural invasion [2]. A small subpopulation of cancer stem cells (CSCs) have been identified in OSCC based on their ability for perpetual self-renewal and proliferation, producing downstream progenitor cells and cancer cells that drive tumor growth $[3,4]$. Therefore, increasing attention is being given to the role of CSCs in the progression of OSCC and their potential as targets for cancer treatment [5]. 
MicroRNAs (miRNAs or miRs), endogenous, and small non-coding single-stranded RNAs of 22 nucleotides that involved in diverse biological processes, including cell cycle, growth, and apoptosis, regulate gene expression by targeting multiple molecules at the posttranscriptional level [6]. Over the past 10 years, many in vivo and in vitro experiments have confirmed the importance of miRNAs in cancer initiation, progression, and metastasis [7]. Moreover, the dysregulation of miRNAs contributes to CSC tumorigenicity, asymmetric cell division, and chemoresistance [8]. Increasing evidence suggests that aberrant expression of miR-495 is associated with the progression of various cancers. A recent study suggests that miR-495 may serve as a tumor suppressor by inhibiting the migration and invasion of gastric cancer cells [9]. In addition, restored miR-495 expression can restrict cell proliferation and invasion in OSCC cells in vitro by repressing its target gene Notch1 [10]. The HOX gene family consists of 39 genes that organize into 4 chromosomal loci, which has been reported to play a significant role in organogenesis via regulation of proliferation, differentiation, survival, migration, and invasion [11]. Homeobox C6 (HOXC6) can be used as a prognostic marker in patients with esophageal squamous cell carcinoma since the median survival time of patients with high HOXC6 expression is found to be poor [12]. Moreover, OSCC patients with lymph node metastasis present with high expression of HOXC6 [13], suggesting the implication of aberrant HOX gene expression in the development of OSCC. Silencing of HOXC6 is capable of inhibiting epithelial-mesenchymal transition (EMT) through the inactivation of the transforming growth factor-beta (TGF- $\beta$ )/Smad signaling pathway in cervical carcinoma cells [14]. TGF- $\beta$ encompasses TGF- $\beta 1,2$, and 3 and has been reported to play a significant role in the regulation of cancer by suppressing tumor growth in the early stage but promoting tumor growth in established cancers [15]. High TGF- $\beta$ expression at tumor center predicts poorer overall survival and recurrence-free and disease-free survival in OSCC patients [16]. However, the reports on the interaction among miR-495, HOXC6, and the TGF- $\beta$ signaling pathway in the development of OSCC remain enigmatic. Therefore, the present study aimed to investigate the role of miR-495 as a novel tumor suppressor and its effect on the cell proliferation, migration, and invasion of CSCs in OSCC, which may provide a novel therapeutic target for the treatment of OSCC in the future.

\section{Materials and methods}

\section{Ethics statement}

The study was conducted under the approval of the Institutional Review Board of Jiangxi Cancer Hospital. Informed written consent was obtained from each participant prior to the study. Animal experiments were conducted under the approval of the Animal Ethics Committee of Jiangxi Cancer Hospital.

\section{Microarray-based gene expression profiling}

The OSCC-related gene expression datasets were retrieved from the Gene Expression Omnibus (GEO) database (https://www.ncbi.nlm.nih.gov/geo/). Then, the GSE31853, GSE74530, and GSE30784 datasets were obtained (all contained different normal control samples and OSCC samples) and used for differential analysis. The samples in the three datasets were subjected to differential analysis by the "limma" package and "input" package of $\mathrm{R}$ language software, with the screening threshold of $|\operatorname{logFC}|>2$ and $p$ value $<0.05$. Dataset GSE37991 was also obtained, which contained 40 normal control samples and 40 OSCC samples. The expression of the screened differentially expressed genes (DEGs) was searched from the dataset, and their expression was drawn as a boxplot using the $\mathrm{R}$ language.

TargetScan database (http://www.targetscan.org/vert_71/), DIANA database (http://diana.imis.athena-innovation.gr/DianaTools/index.php? $r=$ microT_CDS/index), mirDIP database (http://ophid.utoronto.ca/mirDIP/index.jsp\#r), and microRNA.org database (http://34.236.212.39/microrna/home.do) were employed for gene screening. In the four datasets, prediction on the miRNAs regulating HOXC6 was performed by setting "HOXC6" as the input and "human" as the species. The predicted results were screened according to the scores, followed by intersection analysis to identify the study subjects for the follow-up study.

A Venn diagram was constructed using the website (http://bioinformatics.psb.ugent.be/webtools/Venn/), which was used to identify the intersection of the screened results from different datasets. Elements of the different datasets were included in the website, and the names of these datasets were provided. The Venn diagram was constructed using the website, and the intersection of the different datasets was identified.

\section{Purification and identification of OSCC cells}

Sixty cases of fresh OSCC tissue samples were collected after the surgical resection in the maxillofacial surgery of Jiangxi Cancer Hospital (Nanchang, Jiangxi, China) and used for sample selection and pre-treatment. Antipollution treatment for samples was then conducted. In brief, deep tissues of about $1.0 \times 1.0 \times 0.5 \mathrm{~cm}^{3}$ in size were washed with $0.9 \%$ saline containing $250 \mathrm{~mL}$ of 1.6 million units penicillin until the tissues turned white for 3 to 4 times. Then, the tissues were immersed in saline vials, sealed tightly, and sent to the laboratory.

For culture and purification of OSCC cells, the tissues were placed in Petri dishes and cut to expose the fresh tissues. Then, the fresh tissues were rinsed twice with 
sterile phosphate buffer saline (PBS), added with serumfree culture medium, and cut into the size of about 1 $\mathrm{mm}^{3}$ and tissue debris. A total of $3 \mathrm{~mL}$ of tissue and culture medium was transferred into small test tubes and added with collagenase IV, followed by detachment by culture in a $\mathrm{CO}_{2}$ incubator for about $1 \mathrm{~h}$. Then, the detached tissues were centrifuged at $1500 \mathrm{rpm}$ using a lowspeed centrifuge with the supernatant discarded. Next, 3 $\mathrm{mL}$ of culture medium was added to the tissues, and they were repeatedly triturated with a pipette and centrifuged at a low speed, with the supernatant discarded. The procedure was repeated twice. After the addition of $4 \mathrm{~mL}$ of Iscove's modified Dulbecco's medium (IMDM) containing $10 \%$ serum, the cells were seeded into the first two wells of 6-well plates and added with IMDM until the volume reached $3 \mathrm{~mL}$, followed by culture in a $\mathrm{CO}_{2}$ incubator. After $24 \mathrm{~h}$, the adherent cells were detached using $0.25 \%$ trypsin and inoculated. The nonadherent tissues and cells were transferred to the next well every $30 \mathrm{~min}$. The cells from the first two wells that were filled with $3 \mathrm{~mL}$ of IMDM containing 10\% serum were collected and cultured in an incubator after repeated adherence achieved on the 6th wells.

\section{Observation and the change of medium}

After $12 \mathrm{~h}$ of incubation, most of the cells and tissues were firmly adhered to wall and cells expanded and became larger. After $24 \mathrm{~h}$ of culture, the medium was replaced and the non-adherent cells and tissues were gently removed, while the remaining cells were cultured in the new medium.

\section{Repeated adherence and differential culture for a second time}

When cells reached about $80 \%$ confluence, they were treated with trypsin and removed from the third row of wells to a new 6-well plate. After repeated adherence and culture, the relatively purified OSCC cells were obtained.

The identification of OSCC cells was performed. In short, immunohistochemistry was applied to stain keratin, Vimentin, CSC-related identification, and isolation markers BMI1, ALDH1, and CD44. Slides containing OSCC cells were prepared on the strictly sterilized coverslips. The staining was performed following the manufacturer's instructions of the streptavidin peroxidase (SP) immunohistochemistry kit, with polyclonal antibodies purchased from Abcam (Cambridge, UK) used as the primary antibodies: keratin (1:1000, ab155478), Vimentin (1:1000, ab16700), BMI1 (1:1000, ab155478), ALDH1 (1: 1000, ab155478), and CD44 (1:1000, ab155478). Positive and negative controls were prepared during staining. Known positive cell slides were used as the positive control, while PBS was used to replace the primary antibody as the negative control (NC). The cytoplasm and/or cell membrane showing tan-stained particles was considered positive.

\section{Sorting of CSCs in OSCC}

The CSCs at logarithmic growth phase were collected and adjusted to a density of $1 \times 10^{7}$ cells $/ \mathrm{mL}$. Cells were then incubated with anti-CD133-PE (566,593, 1:500, Becton, Dickinson and Company, NJ, USA) (with absorbed light of 490-560 $\mathrm{nm}$ and emission wavelength of $595 \mathrm{~nm}$ ) and anti-CD44-fluorescein isothiocyanate (FITC) $(555,478,1: 500$, Becton, Dickinson and Company, NJ, USA) (with excitation wavelength of $495 \mathrm{~nm}$ and emission wavelength of $519 \mathrm{~nm}$ ) for $30 \mathrm{~min}$ at room temperature. The cells were subsequently resuspended in $1 \mathrm{~mL}$ of PBS, filtered through a $40-\mu \mathrm{m}$ aseptic screen mesh and placed on ice for analyzing and sorting. The isotype control antibody was used to label cells under the same conditions. Cells were then sorted using a FACSAria II flow cytometer (Becton, Dickinson and Company, NJ, USA) for detection, followed by fluorescence labeling using different antibodies, while the CD133 and CD44 double-positive cells to be sorted were circled. Lastly, the circled cells were sorted aseptically into sorting tubes.

\section{CSC suspension sphere-forming test}

The sorted CD133 and CD44 double-positive cells were seeded into low-adsorptive 96-well plates and cultured with serum-free Dulbecco's modified Eagle's medium (DMEM)-F12 containing $20 \mathrm{ng} / \mathrm{mL}$ epithelial growth factor (EGF) and $20 \mathrm{ng} / \mathrm{mL}$ fibroblast growth factor (FGF)$\beta$ for 10 consecutive days, with the medium changed semi-quantitatively every 2 days. Lastly, the cells were observed, photographed, and counted under a CKX41 inverted optical microscope (Olympus Optical Co., Ltd., Tokyo, Japan).

\section{Dual-luciferase reporter assay}

The Bioinformatics Database (microRNA.Org; http: //www.microrna.org/) was employed to predict whether HOXC6 was a target gene of miR-495. Human embryonic kidney HEK293T cells were cultured in DMEM containing $10 \%$ fetal bovine serum (FBS) with $5 \% \mathrm{CO}_{2}$ at $37^{\circ} \mathrm{C}$. The complementary DNA (cDNA) fragment of HOXC6 3 '-untranslated region ( 3 '-UTR) containing the miR-495 binding site was inserted into the pmirGLO vector. The cDNA fragment of HOXC6 3'-UTR with the mutated binding site was constructed by sitedirected mutagenesis and inserted into the pmirGLO vector. The pmirGLO-HOXC6 (WT) or pmirGLOmutant type (Mut) HOXC6 recombinant vector was then co-transfected into HEK293T cells with miR-495 mimic (miR-495 overexpression sequence) or miR-NC (NC sequence) by lipofection. After $48 \mathrm{~h}$ of culture, the 
cells were collected and lysed. An amount of $100 \mu \mathrm{L}$ of Renilla luciferase detection working solution was added to $100 \mu \mathrm{L}$ of lysate supernatant, followed by the detection of Renilla luciferase activity. In addition, $100 \mu \mathrm{L}$ of firefly luciferase detection reagent was mixed with $100 \mu \mathrm{L}$ of lysate supernatant before the detection of firefly luciferase activity. A multi-functional microplate reader SpectraMax M5 was used to assess the activity of Renilla luciferase and firefly luciferase, respectively, with interval time set as $2 \mathrm{~s}$ and the detection time as $10 \mathrm{~s}$.

\section{Cell treatment}

The CSCs were cultured in high-glucose DMEM containing $10 \% \mathrm{FBS}$ in a $5 \% \mathrm{CO}_{2}$ incubator at $37^{\circ} \mathrm{C}$ with saturated humidity. The cells were sub-cultured when cells reached approximately $75 \%$ confluence. The cells in logarithmic growth phase were treated with trypsin and added with $1.4 \mathrm{~mL}$ of PBS to adjust cell density to $2 \times$ $10^{5}$ cells $/ \mathrm{mL}$. Cells were then assigned into 6 groups: blank (CSCs without transfection), NC (CSCs transfected with scramble small interfering RNA [siRNA]), miR-495 mimic (CSCs transfected with miR-495 mimic, purchased from Invitrogen Inc., Carlsbad, CA, USA), miR-495 inhibitor (CSCs transfected with miR-495 inhibitor, purchased from Sigma-Aldrich Chemical Company, St Louis, MO, USA), si-HOXC6 (CSCs transfected with silenced HOXC6, purchased from Shanghai GenePharma Co., Ltd., Shanghai, China), and miR-495 inhibitor + si-HOXC6 (CSCs transfected with miR-495 inhibitor and added with $100 \mathrm{ng} / \mathrm{mL}$ silenced HOXC6 to stimulate cells). The sequences of transfected primers are listed in Table 1. After $6 \mathrm{~h}$ of transfection, the medium was changed, after which cells were then incubated for $48 \mathrm{~h}$, and collected for subsequent experiments. Human OSCC cells in logarithmic growth phase were seeded into 6-well plates. When cells reached about $30-50 \%$ confluence, cells were transfected according to the manufacturer's instructions of lipofectamine 2000 (Invitrogen, Carlsbad, CA, USA). About $100 \mathrm{pmol}$ of miR-495 mimic, miR-495 inhibitor, si-HOXC6, and their NCs (final concentration was $50 \mathrm{nM}$ ) were diluted in $250 \mu \mathrm{L}$ of serum-free Opti-minimum essential medium (MEM) (Gibco, Grand Island, NY, USA), mixed well, and incubated at room temperature for $5 \mathrm{~min}$.

Table 1 Sequences of transfection primers

\begin{tabular}{ll}
\hline Groups & Primer sequences (5'-3') \\
\hline siRNA-NC & UUCUUCUAACUUUUCACGUUC \\
miR-495 mimic & AAAGAACACTITTCGGTATT \\
miR-495 inhibitor & AATACCGAAAAGTGTTCTTT \\
SiRNA-HOXC6 & UUUAAUAUUUAUUUCUGUCUC
\end{tabular}

siRNA small interfering RNA, HOXC6 homeobox $\mathrm{C} 6, N C$ negative control, miR-495 microRNA-495
Next, $5 \mu \mathrm{L}$ of lipofectamine 2000 was mixed with $250 \mu \mathrm{L}$ of serum-free medium Opti-MEM and incubated for 5 min at room temperature. The above two solutions were then mixed together and incubated at room temperature for $20 \mathrm{~min}$, followed by addition to the cell culture wells. After incubation at $37^{\circ} \mathrm{C}$ with $5 \% \mathrm{CO}_{2}$ for $6-8 \mathrm{~h}$, cells were then incubated with DMEM containing 10\% FBS and penicillin/streptomycin for $24-48 \mathrm{~h}$ before subsequent experiments.

\section{Reverse transcription-quantitative polymerase chain reaction}

Total RNA was extracted from cells of each group using RNA extraction kit (Invitrogen, Carlsbad, CA, USA), followed by determination of RNA concentration. Primers for miR-495, HOXC6, TGF- $\beta$, TGF $\beta$ RI, TGFßRII, Smad4, Vimentin, E-cadherin, N-cadherin, glyceraldehyde phosphate dehydrogenase (GAPDH), and U6 were designed and synthesized by Takara (Takara Holdings Inc., Kyoto, Japan) (Table 2). Then, the total RNA was reverse transcribed into cDNA according to the manufacturer's instructions of reverse transcription kit (DRR047S, TaKaRa Co., Ltd., Dalian, China). RTqPCR was conducted using ABI 7500 instrument (ABI Company, Oyster Bay, NY). U6 was used as an internal reference for the relative expression of miR-495, while

Table 2 The primer sequences for RT-qPCR

\begin{tabular}{|c|c|}
\hline Gene & Sequence $\left(5^{\prime}-3^{\prime}\right)$ \\
\hline miR-495 & $\begin{array}{l}\text { Forward: GCGCGTGAGCAGGCTGGAGAAATT } \\
\text { Reverse: AAACAAACATGGTGCA }\end{array}$ \\
\hline HOXC6 & $\begin{array}{l}\text { Forward: ACAGACCTCAATCGCTCAGGA } \\
\text { Reverse: AGGGGTAAATCTGGATACTGGC }\end{array}$ \\
\hline TGF- $\beta$ & $\begin{array}{l}\text { Forward: CAACGCCATCTATGAGAAAACC } \\
\text { Reverse: AAGCCCTGTATTCCGTCTCC }\end{array}$ \\
\hline TGF $\beta R I$ & $\begin{array}{l}\text { Forward: ATTACCTGGACATCGGCA AC } \\
\text { Reverse: TTGGGCACCACATCATAGAA }\end{array}$ \\
\hline Smad4 & $\begin{array}{l}\text { Forward: GTGGCTGGTCGGAAAGGATT } \\
\text { Reverse: ACTGGCAGGCTGACTTGTGG }\end{array}$ \\
\hline TGF $\beta R \|$ & $\begin{array}{l}\text { Forward: ACTTGACCTGTTGCCTGTGTGAC } \\
\text { Reverse: CTGGCTTCAACGCCTITCACCTCA }\end{array}$ \\
\hline $\mathrm{N}$-cadherin & $\begin{array}{l}\text { Forward: TCATTGCCATCCTGCTCTGCAT } \\
\text { Reverse: AGTTGTTTGCCTGGCGTTCTT }\end{array}$ \\
\hline Vimentin & $\begin{array}{l}\text { Forward: AAAGTGTGGCTGCCAAGAACCT } \\
\text { Reverse: ATTCACGCATCTGGCGTTCCA }\end{array}$ \\
\hline E-cadherin & $\begin{array}{l}\text { Forward: TTAGGTTAGAGGGTTATCGCGT } \\
\text { Reverse: TAACTAAAAATTCACCTACCGACC }\end{array}$ \\
\hline U6 & $\begin{array}{l}\text { Forward: CGCTTCACGAATTTGCGTGTCAT } \\
\text { Reverse: GCTTCGGCAGCACATATACTAAAAT }\end{array}$ \\
\hline GAPDH & $\begin{array}{l}\text { Forward: ACAGTCAGCCGCATCTTCTT } \\
\text { Reverse: GACAAGCTTCCCGTTCTCAG }\end{array}$ \\
\hline
\end{tabular}

$R T-q P C R$ reverse transcription quantitative polymerase chain reaction, miR-495 microRNA-495, HOXC6 homeobox C6, TGF- $\beta$ transforming growth factor $\beta$, TGF- $\beta R$ I transforming growth factor $\beta$ receptor I, TGF- $\beta R / l$ transforming growth factor ßreceptor II, Smad4 sma and mother against ddp four, GAPDH glyceraldehyde-3-phosphate dehydrogenase, U6 U6 snRNA 
GAPDH was used as an internal reference for the relative expression of HOXC6, TGF- $\beta$, TGF $\beta$ RI, TGF $\beta$ RII, Smad4, Vimentin, E-cadherin, and N-cadherin. The fold changes were calculated using the relative quantification $\left(2^{-\Delta \Delta \mathrm{Ct}}\right.$ method) [17].

\section{Western blot analysis}

Total protein was extracted from cells and tissues using radio-immunoprecipitation assay (RIPA) lysis buffer (Gibco, Carlsbad, CA, USA) containing phenylmethanesulfonyl fluoride (PMSF), and protein concentration was determined using the Bradford method. After separation with $10 \%$ sodium dodecyl sulfate-polyacrylamide gel electrophoresis (SDS-PAGE), the proteins were subsequently transferred onto polyvinylidene fluoride (PVDF) membranes. The membranes were blocked with Trisbuffered saline with Tween 20 (TBST) containing 5\% skim milk powder for $1 \mathrm{~h}$, and then incubated overnight at $4{ }^{\circ} \mathrm{C}$ with the following primary antibodies: CD133 (1: 500, ab19898, Abcam), CD44 (1:2000, ab157107, Abcam), HOXC6 (1:1000, ab191542, Abcam), TGF- $\beta$ (1: 1000, ab190503, Abcam), TGF $\beta$ RI (1:1000, ab31013, Abcam), TGF $\beta$ RII (1:1000, ab78419, Abcam), Smad2 (1: 1000, 5339, Cell Signaling Technology), Smad4 (1:5000, ab40759, Abcam), Smad7 (1:1000, ab90086, Abcam), Ncadherin (1:1000, ab18203, Abcam), Vimentin (1:1000, ab92547, Abcam), Ecadherin (1:50, ab1416, Abcam), and GAPDH (internal reference, 1:5000, Santa Cruz Biotechnology Inc., CA, USA). Subsequently, the membrane was incubated with horseradish peroxidase-labeled secondary antibody at room temperature for $2 \mathrm{~h}$. Finally, the membrane was developed using autoradiography, photographed, and recorded, after which the film was scanned. Quantification analysis was performed using Gel-Pro Analyzer 4.0, and the ratio of the gray value of target bands to that of internal reference bands served as the relative expression of proteins.

\section{3-(4,5-Dimethylthiazol-2-yl)-2,5-diphenyltetrazolium bromide (MTT) assay}

The isolated and purified CSCs were seeded into 96-well plates and grouped into the blank, NC, miR-495 mimic, miR-495 inhibitor, si-HOXC6, and miR-495 inhibitor + si-HOXC6 groups. Cells in each well were added with $20 \mu \mathrm{L}$ of MTT $(5 \mathrm{mg} / \mathrm{mL})$ at $0,24,48$, and $72 \mathrm{~h}$, respectively, followed by $4 \mathrm{~h}$ of incubation at $37^{\circ} \mathrm{C}$ after which the supernatant was discarded. Then, $200 \mu \mathrm{L}$ of dimethyl sulfoxide (DMSO) was added to the cells to dissolve the bluish-violet precipitate. The optical density (OD) value was then measured at $570 \mathrm{~nm}$.

\section{Scratch test}

The effect of miR-495 expression on CSC migration was evaluated by scratch test. After $24 \mathrm{~h}$ of transfection, the cells were seeded into 6-well plates. Once cells reached full confluence, a $10-\mu \mathrm{L}$ pipette tip was used to gently scratch along the central axis of the plate and the floating cells were washed away with PBS. After $24 \mathrm{~h}$ of culture, the cells were observed and photographed under a microscope and the cell migration was expressed as the healing percentage of scratched cells. The percentage of cell healing $=($ scratch width before experiment scratch width after culture for $24 \mathrm{~h}$ )/scratch width before experiment $\times 100 \%$.

\section{Transwell assay}

Basement membranes of the Transwell chambers were coated using Matrigel. The cells in each group were collected after $24 \mathrm{~h}$ of transfection and resuspended in serum-free medium for $16 \mathrm{~h}$ to allow cell starvation. The apical chamber was added with cell suspension at a density of $1 \times 10^{5}$, followed by the addition of culture medium containing $10 \%$ FBS to the basolateral chamber. After $24 \mathrm{~h}$ of incubation in a $5 \% \mathrm{CO}_{2}$ incubator at $37^{\circ} \mathrm{C}$, cells failing to invade were discarded, while the remaining cells were fixed with $4 \%$ paraformaldehyde and stained with $0.5 \%$ crystal violet. At last, the number of cells passing through the membrane was calculated in five randomly selected visual fields under a microscope, with the average value obtained.

\section{Flow cytometry}

Flow cytometry was used to detect the effect of miR-495 on the apoptosis of CSCs. Cell apoptosis was detected by Annexin V-FITC/propidium iodide (PI) double-staining cell apoptosis detection kit (40303ES20, Yeasen Biotechnology Co. Ltd., Shanghai, China). Annexin V+ and PIcells were considered as apoptotic cells. The percentage of early apoptotic cells $(\%)=$ Annexin V-FITC positive cells/total cells $\times 100 \%$.

The cells in the blank group and the miR-495 mimic group after $24 \mathrm{~h}$ of treatment were centrifuged, collected, washed three times with pre-cooled PBS, and centrifuged at $1000 \mathrm{rpm}$ for $5 \mathrm{~min}$ at room temperature with the supernatant discarded. Cells in the tube were added with $1 \mathrm{~mL}$ of pre-cooled $70 \%$ ethanol and fixed overnight at $4{ }^{\circ} \mathrm{C}$. The next day, cells were centrifuged at $700 \mathrm{rpm}$ for $5 \mathrm{~min}$ at room temperature, resuspended in $0.5 \mathrm{~mL}$ of PBS, added with RNase A (final concentration of $50 \mu \mathrm{g} / \mathrm{mL}$ ), and allowed to stand for $60 \mathrm{~min}$ at room temperature. Cells were then stained with $500 \mu \mathrm{L}$ of PI $(100 \mu \mathrm{g} / \mathrm{mL})$ and incubated for $30 \mathrm{~min}$ in the dark. CellQuest was finally used to detect cells at the G0/G1, S, and $\mathrm{G} 2 / \mathrm{M}$ phases.

\section{Xenograft tumor in nude mice}

A total of 36 female-specific pathogen-free (SPF) grade BALB/C nude mice (4-6 weeks old) (Animal Center of 
Sichuan University, Chengdu, Sichuan, China) were used in this study. The feeding of nude mice and all animal experiments were performed in the animal center. The successfully constructed lentiviral vectors were subcutaneously injected into nude mice using a 1-mL syringe. The nude mice were assigned into the blank (without lentiviral vector injection), NC (injected with NC lentiviral vector), miR-495 agomir (injected with miR-495 agomir lentiviral vector), miR-495 antagomir (injected with miR-495 antagomir lentiviral vector), short hairpin RNA (sh)-HOXC6 (injected with sh-HOXC6 lentiviral vector), and miR-495 inhibitor + sh-HOXC6 (injected with miR-495 inhibitor and sh-HOXC6 lentiviral vector) groups. After inoculation of the lentiviral vector, all nude mice were fed in the laminar hood of SPF animal room. The long diameter $(L)$ and short diameter $(W)$ of the tumor were measured every 7 days. The tumor volume $(V)=1 / 2 \times L W 2$ was calculated, and the tumor growth curve was drawn. On the 28th day, the nude mice were euthanized using cervical dislocation method and their xenografts were removed for photographing and comparison.

\section{Statistical analysis}

Statistical analyses were conducted using the SPSS 21.0 statistical software (IBM Corp. Armonk, NY, USA). Measurement data in accordance with normal distribution were expressed as mean \pm standard deviation. Data between two groups were compared by independent sample $t$ test, and data among multiple groups were compared by one-way analysis of variance (ANOVA). Pairwise comparisons were conducted by least significant difference (LSD) test. A value of $p<0.05$ was considered statistically significant.

\section{Results}

\section{Bioinformatics analysis predicting the DEGs and their} molecular interactions in OSCC

OSCC-related sequencing datasets were retrieved from the GEO database, and three datasets, GSE31853, GSE74530, and GSE30784, were selected. Next, the 3 datasets were subjected to differential analysis, and 284, 374, and 420 OCSS-associated DEGs were found in the GSE31853, GSE74530, and GSE30784 datasets, respectively (Fig. 1a-c). To further screen the OSCC-associated genes, Venn diagrams were constructed by including the top 100 DEGs from the 3 datasets (Fig. 1d). HOXC6 was found to be the only gene that presented in the top 100 DEGs from the 3 datasets, suggesting the potential association of HOXC6 with OSCC. Analysis of HOXC6 expression in these 3 datasets revealed that HOXC6 was highly expressed in OSCC. In order to further confirm the expression of HOXC6 in OSCC, HOXC6 expression was analyzed in the GSE37991 dataset, which also showed the significantly elevated HOXC6 expression in OSCC tissues (Fig. 1e). This result further suggested that HOXC6 was likely to promote OSCC development. Previous studies of OSCC-related signaling pathways revealed that the TGF- $\beta$ signaling pathway was closely related to OSCC development $[18,19]$. However, little is known about whether HOXC6 regulates the TGF- $\beta$ signaling pathway in OSCC. In order to further understand the mechanism of HOXC6 in OSCC, the potential regulatory miRNAs of HOXC6 were predicted using the TargetScan database, in which the predicted results with "context ++ score percentile" greater than 90 were selected. Based on the predicted results from DIANA database, the scores greater than 0.8 were selected. For prediction using mirDIP, the predicted results with a score greater than 0.5 were selected. Based on the microRNA.org database, a total of 24 regulatory miRNAs were predicted and included for the follow-up analysis. Venn analysis was then conducted for the aforementioned 24 miRNAs and depicted (Fig. 1f) 4 intersected miRNAs, namely hsa-miR-377, hsa-miR-27a, hsa-miR-27b, and hsa-miR-495. Previous studies have highlighted the functional mechanisms of hsa-miR-377, hsa-miR-27a, and hsa-miR-27b in OSCC [20-24], but the studies on the mechanism of hsa-miR-495 in OSCC were limited.

Furthermore, analysis using online bioinformatic software revealed a specific binding region between HOXC6 gene sequence and miR-495 sequence, suggesting that HOXC6 was a target gene of miR-495 (Fig. 1g). Dualluciferase reporter assay confirmed the binding of miR495 to HOXC6. As shown in Fig. 1h, compared with the NC group, the miR-495 mimic group showed a significant decrease in the luciferase activity of HOXC6 wild type $(\mathrm{Wt})$ in cells $(p<0.05)$ while there was no significant difference in the luciferase activity of HOXC6-Mut $(p>0.05)$, suggesting that miR-495 could specifically bind to HOXC6. Based on the preliminary analysis from this study, we found that hsa-miR-495 was likely to regulate the expression of HOXC6, thereby affecting the TGF- $\beta$ signaling pathway and the progression of OSCC.

\section{Successful isolation of OSCC cells}

The relatively purified OSCC cells were obtained by repeated adherence and differential culture for 5 days. Morphological observation results showed that the OSCC cells were tetragonal or polygonal resembling "paving stones" (Additional file 1: Figure S1A). Purified cells were identified by Vimentin and keratin staining using immunohistochemistry. Vimentin was negatively expressed in OSCC cell slides (Additional file 1: Figure S1B) and keratin was positively expressed in OSCC slides (Additional file 1: Figure S1C), suggesting that the purified OSCC cells were 


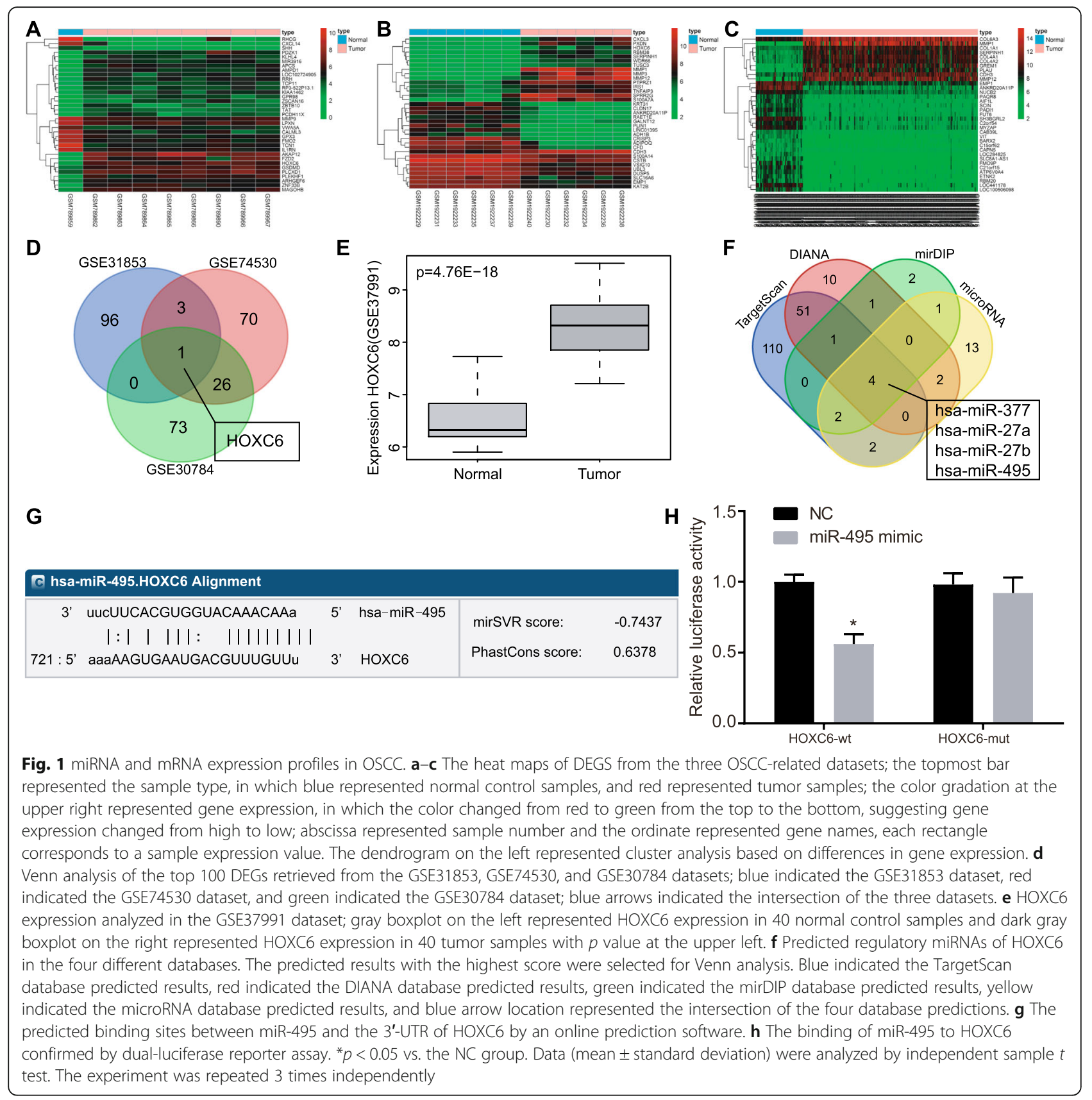

successfully obtained. The OSCC cells were then sorted and identified.

\section{Successful identification of CSCs}

Using flow cytometry, CD133 and CD44 positive and negative cells were sorted from OSCC cells (Additional file 2: Figure S2A). Then, the cell spheres enriched in OSCC cells were treated with CSC suspension sphere-forming test in order to identify whether the cell spheres were the CSCs (Additional file 2: Figure S2B). Subsequently, Western blot analysis was performed to detect the expression of stem-related surface molecular markers CD133 and CD44 in OSCC cell spheres, the results of which displayed that the expression of CD133 and CD44 was significantly increased in OSCC cell spheres compared with normal OSCC cells $(p<0.05)$ (Additional file 2: Figure S2C, D). These findings suggested that CSCs were successfully isolated.

\section{miR-495 inhibits activation of the TGF- $\beta$ signaling pathway by downregulating HOXC6}

RT-qPCR and Western blot analysis were used to measure the levels of miR-495, HOXC6, TGF- $\beta$, TGF $\beta$ RI, TGF $\beta$ RII, Smad2, and Smad4 in cells. The results of RT- 
qPCR are depicted in Fig. 2a. There was no significant difference in miR-495 level and the mRNA level of HOXC6, TGF- $\beta$, TGF $\beta$ RI, TGF $\beta$ RII, Smad2, and Smad4 between the blank and NC groups $(p>0.05)$. Compared with the blank and NC groups, the mRNA levels of HOXC6, TGF- $\beta$, TGF $\beta$ RI, TGF $\beta$ RII, Smad2, and Smad4 were significantly lower in the miR-495 mimic group while the expression of miR-495 was higher $(p<0.05)$. Similarly, the levels of HOXC6, TGF- $\beta$, TGF $\beta$ RI, TGFßRII, Smad2, and Smad4 were obviously decreased in the si-HOXC6 group compared to the blank and NC groups $(p<0.05)$, while miR-495 level did not differ significantly $(p>0.05)$. The miR-495 inhibitor group exhibited markedly higher mRNA levels of TGF- $\beta$, TGF $\beta$ RI, TGFßRII, Smad2, and Smad4 yet lower miR-495 level in comparison with the blank and NC groups $(p<0.05)$. In the miR-495 inhibitor + si-HOXC6 group, there was no significant difference in the mRNA levels of HOXC6, TGF- $\beta$, TGF $\beta$ RI, TGF $\beta$ RII, Smad2, and Smad4 $(p>$ $0.05)$, while miR-495 level was remarkably decreased $(p<0.05)$ compared to the blank and NC groups.

Western blot analysis displayed no significant difference in the protein levels of HOXC6, TGF- $\beta$, TGF $\beta$ RI, TGFBRII, Smad2, and Smad4 as well as the extent of Smad2 and Smad4 phosphorylation between the blank group and the NC group $(p>0.05)$. In comparison with the blank and NC groups, the protein levels of HOXC6, TGF- $\beta$, TGF $\beta$ RI, TGF $\beta$ RII, Smad2, and Smad4 as well as the extent of Smad2 and Smad4 phosphorylation were diminished in the miR-495 mimic and si-HOXC6 groups (all $p<0.05$ ) while an opposite trend was observed in the
miR-495 inhibitor group (all $p<0.05$ ). The miR-495 inhibitor + si-HOXC6 group showed no changes compared to the blank and NC groups $(p>0.05)$ (Fig. 2b, c). These results demonstrated that the upregulation of miR-495 or downregulation of HOXC6 could inhibit the expression of the TGF- $\beta$ signaling pathway-related genes in CSCs following OSCC.

\section{Overexpression of miR-495 or silencing of HOXC6 inhibits EMT in CSCs}

Western blot analysis was carried out to determine the effect of miR-495 and HOXC6 on EMT in CSCs. The results (Fig. 3) showed no significant difference regarding the EMT between the blank group and the NC group $(p>0.05)$. Compared with the blank and $\mathrm{NC}$ groups, the miR-495 mimic and si-HOXC6 groups displayed an obvious reduction in the levels of $\mathrm{N}$-cadherin and Vimentin, while a significant increase in E-cadherin level $(p<0.05)$. The miR-495 inhibitor group showed a remarkable increase in the levels of $\mathrm{N}$-cadherin and Vimentin but a reduced E-cadherin level was observed $(p<0.05)$. No evident significance in the EMT was found in the miR-495 inhibitor + si-HOXC6 group relative to the blank and NC groups $(p>0.05)$. The above results led to a conclusion that the overexpression of miR-495 or knockdown of HOXC6 could obstruct EMT in CSCs.

Overexpression of miR-495 or silencing of HOXC6 inhibits migration, invasion, and proliferation of CSCs in OSCC

Scratch test was performed to detect cell migration in each group, and the results demonstrated that there was

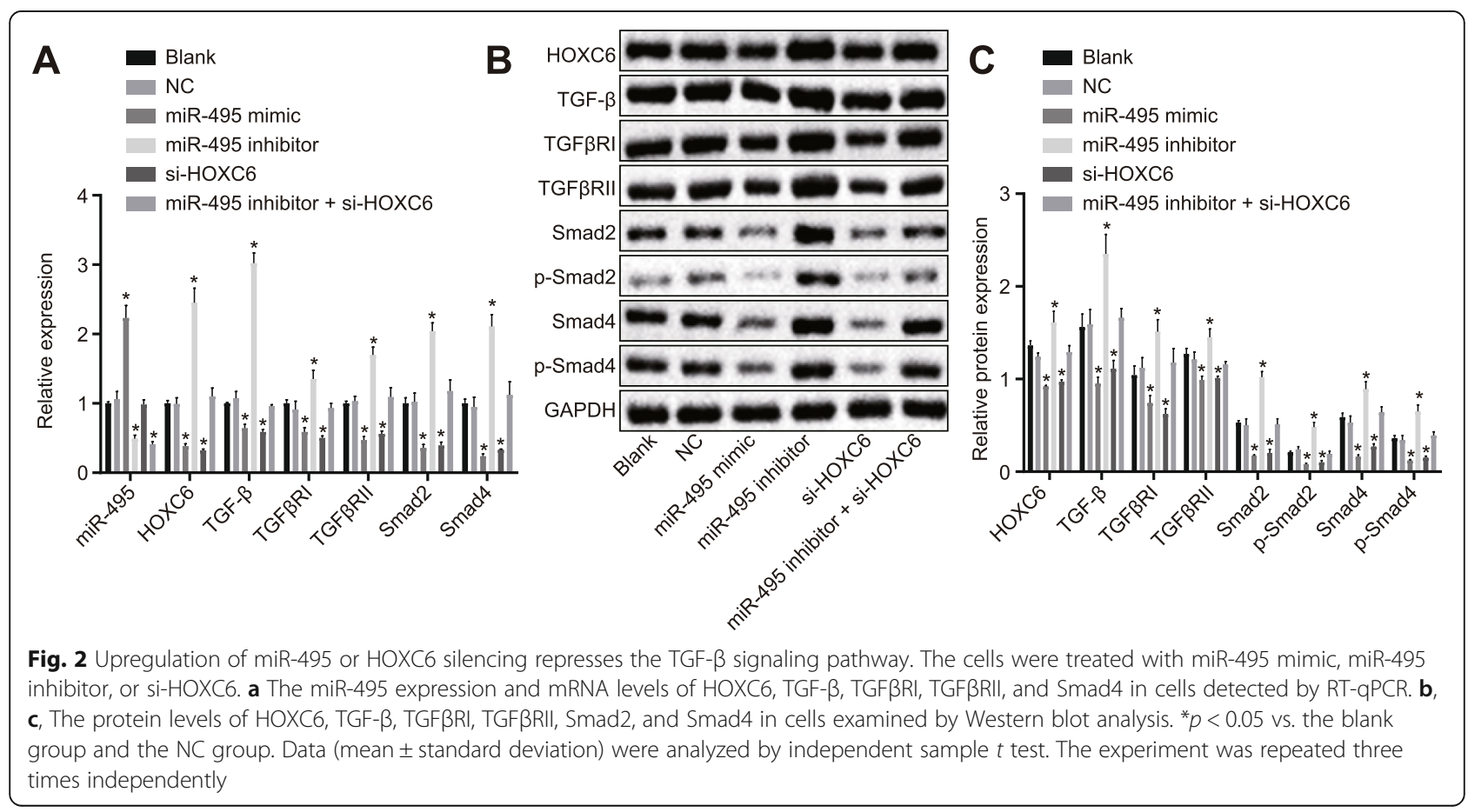



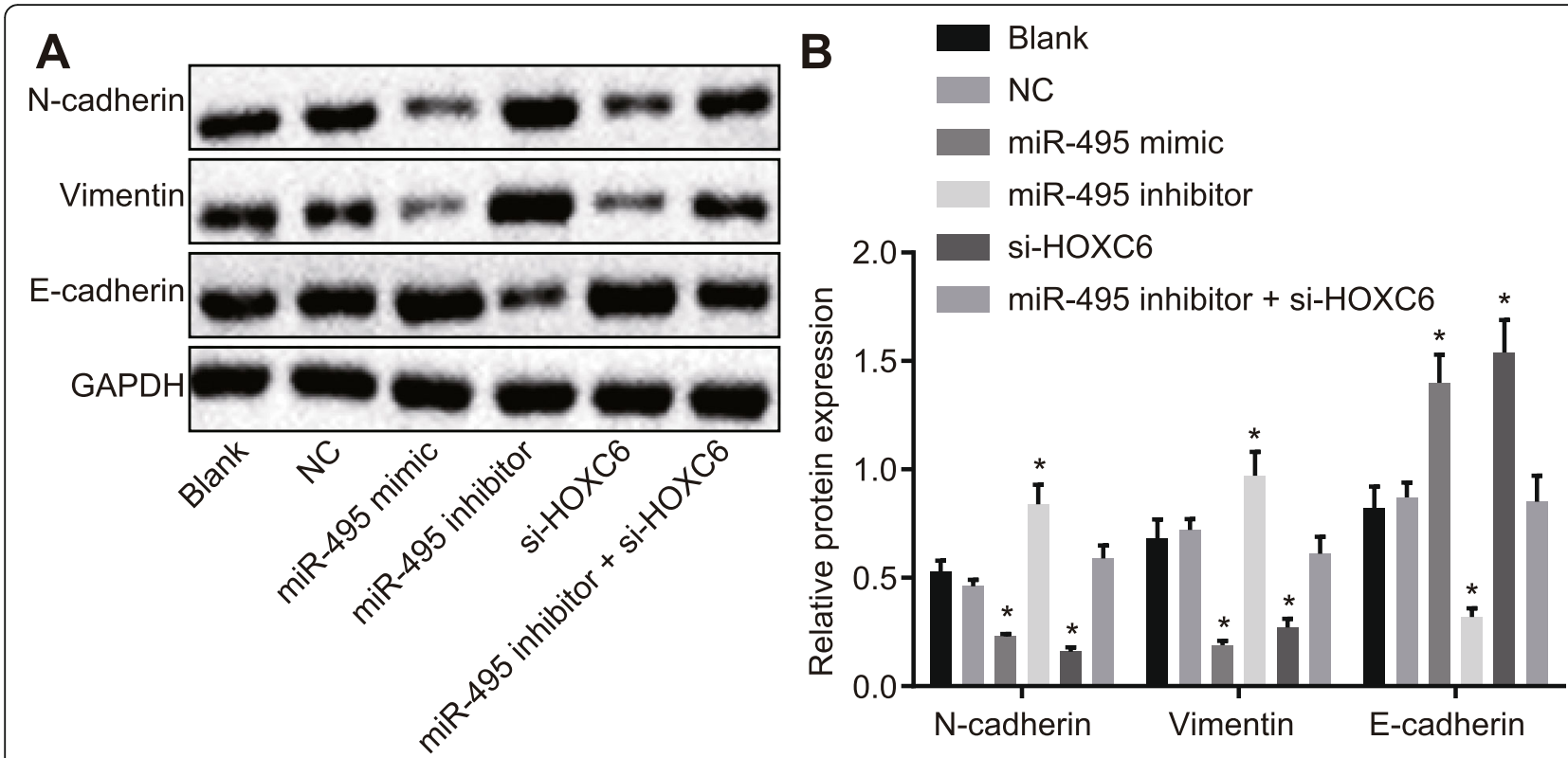

Fig. 3 Upregulation of miR-495 or HOXC6 silencing inhibits EMT in CSCs. The cells were treated with miR-495 mimic, miR-495 inhibitor, or siHOXC6. a, b The protein expression of N-cadherin, Vimentin, and E-cadherin in CSCs determined by Western blot analysis. ${ }^{*} p<0.05$ vs. the blank and NC groups. Data (mean \pm standard deviation) were analyzed by independent sample $t$ test. The experiment was repeated three times independently

no pronounced difference in cell migration between the blank group and the NC group $(p>0.05)$. Compared with the NC and blank groups, the miR-495 mimic and si-HOXC6 groups revealed a significantly reduced cell migration $(p<0.05)$, while the miR-495 inhibitor group exhibited a significantly enhanced cell migration $(p<$ $0.05)$. However, cell migration did not change remarkably in the miR-495 inhibitor + si-HOXC6 group $(p>$ 0.05) (Fig. 4a, b).

Transwell assay was performed to test cell invasion in each group, and the results demonstrated that there was no remarkable difference in cell invasion between the blank group and the NC group $(p>0.05)$. Compared with the NC and blank groups, the miR-495 mimic and si-HOXC6 groups displayed a significantly reduced cell invasion $(p<0.05)$, while the miR-495 inhibitor group displayed a significantly elevated cell migration $(p<$ 0.05). However, the miR-495 inhibitor + si-HOXC6 group showed no significant changes in cell migration compared with the NC and blank groups $(p>0.05)$ (Fig. 4c, d).

MTT assay was employed to examine the proliferation of CSCs in each group. The results (Fig. 4e) demonstrated that CSC proliferation in each group showed no obvious changes at $0 \mathrm{~h}(p>0.05)$. Compared with the cell proliferation at $0 \mathrm{~h}$, the cell proliferation changed remarkably at $24 \mathrm{~h}, 48 \mathrm{~h}$, and $72 \mathrm{~h}(p<0.05)$. Compared with the blank and NC groups, the miR-495 inhibitor group demonstrated significantly faster cell proliferation $(p<0.05)$, while the miR-495 mimic and si-HOXC6 groups showed a significantly slower cell proliferation $(p<0.05)$. The miR-495 inhibitor + si-HOXC6 group exhibited no significant difference in cell proliferation when compared to the blank and NC groups $(p>0.05)$. The above results showed that upregulation of miR-495 or silencing of HOXC6 could inhibit cell proliferation. In sum, overexpression of miR-495 or silencing of HOXC6 exerted an inhibitory effect on CSC cell migration, invasion, and proliferation.

\section{Overexpression of miR-495 prevents cell cycle entry and} promotes CSC apoptosis by suppressing HOXC6

PI staining was conducted to determine cell cycle distribution in each group. The results (Fig. 5a, b) showed that there was no pronounced difference in the cell cycle between the blank group and the NC group $(p>0.05)$. Compared with the blank and NC groups, the miR-495 mimic and si-HOXC6 groups showed more cells arrested in the G1 phase, while fewer in the $\mathrm{S}$ phase $(p<$ 0.05), demonstrating that the proliferation of CSCs was significantly inhibited. However, there were fewer cells arrested in the G1 phase and more cells arrested in the $\mathrm{S}$ phase in the miR-495 inhibitor group compared to the blank and NC groups $(p<0.05)$. No significant difference was detected in cells arrested in the $\mathrm{G} 1$ and $\mathrm{S}$ phases among the blank, NC, and miR-300 inhibitor + siHOXC6 groups $(p>0.05)$.

Annexin V/PI double staining was employed to examine cell apoptosis. The results (Fig. $5 \mathrm{c}$, d) revealed that there was no marked difference in cell apoptosis 


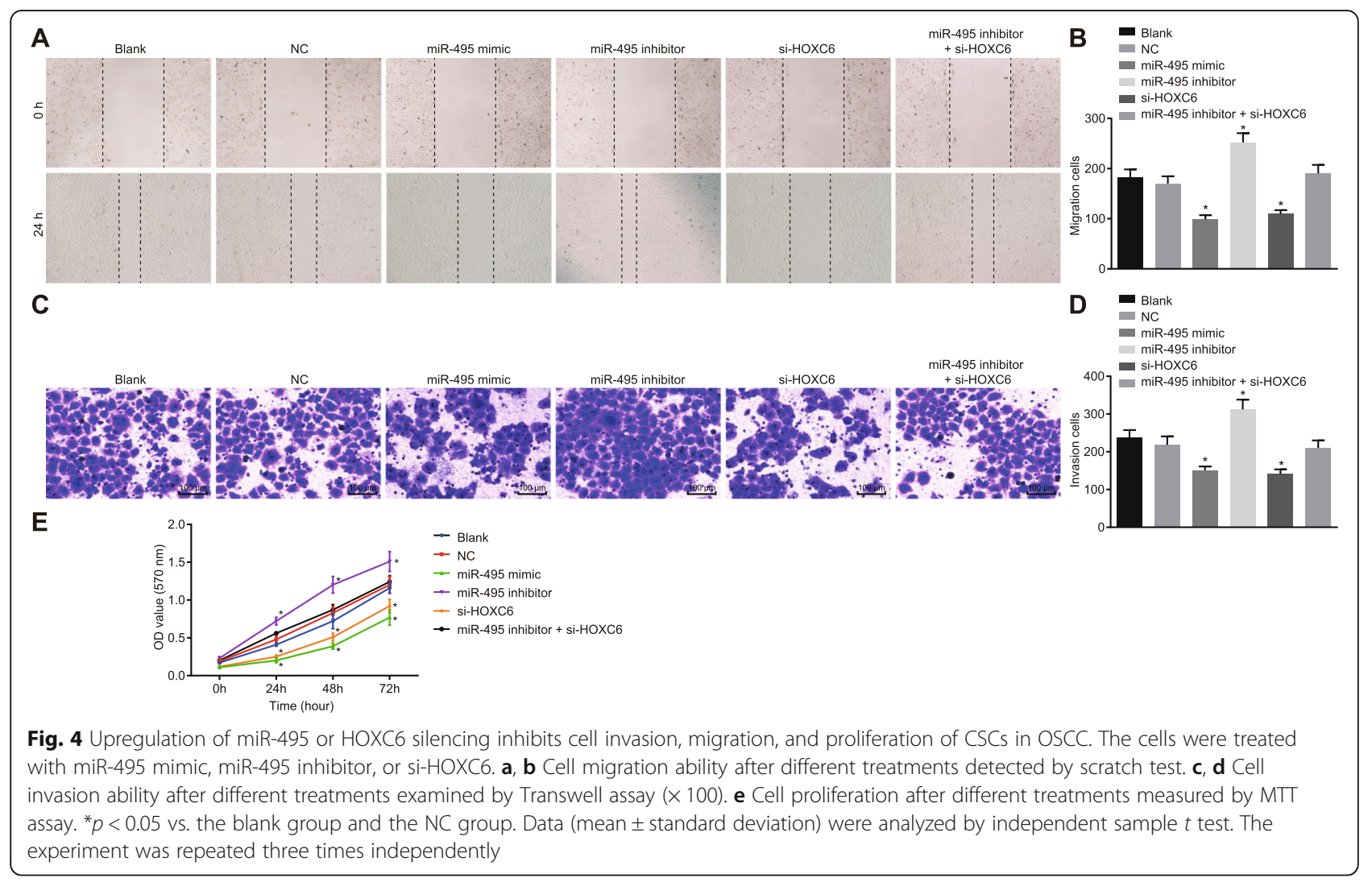

between the blank group and the NC group $(p>0.05)$. Compared with the blank and NC groups, the cell apoptosis was significantly decreased in the miR-495 inhibitor group $(p<0.05)$, while that was markedly enhanced in the miR-495 mimic and si-HOXC6 groups $(p<0.05)$. However, there was no difference in cell apoptosis among the blank, NC, and miR-495 inhibitor + siHOXC6 groups $(p>0.05)$. These results suggested that upregulated miR-495 could suppress cell cycle entry and promote cell apoptosis in CSCs by suppressing HOXC6.

\section{Overexpression of miR-495 or silencing of HOXC6 represses tumor growth in vivo}

Xenograft tumor in nude mice was used to determine the effects of miR-495 and HOXC6 on the tumor growth in vivo. The tumor growth curve illustrated (Fig. 6) that compared with the NC and blank groups, the miR-495 agomir and sh-HOXC6 groups showed delayed tumor growth, while the miR-495 antagomir group demonstrated boosted tumor growth of nude mice after 2 weeks of treatment, with a progressive increase as time went by $(p<0.05)$. After 4 weeks, the mice were euthanized and the tumors were extracted and measured. Compared with the blank and NC groups, the miR-495 agomir and sh-HOXC6 groups displayed significantly decreased tumor volume and weight $(p<0.05)$. The tumor volume and weight were increased in the miR-
495 antagomir group in comparison with the blank and NC groups $(p<0.05)$. However, no obvious difference regarding tumor volume and weight was observed in the blank, NC, and miR-495 antagomir + sh-HOXC6 groups $(p>0.05)$. These results showed that miR-495 overexpression could suppress tumor growth in vivo by inhibiting HOXC6.

\section{Successful transfection of miR-495 interference plasmids}

RT-qPCR was used to detect the transfection efficiency of miR-495 overexpression and knockdown. The results (Additional file 3: Figure S3) showed that compared with the NC-mimic group, the expression of miR-495 was increased in the miR-495 mimic group $(p<0.05)$. The expression of miR-495 was lower in the miR-495 inhibitor group than that in the NC-inhibitor group $(p<0.05)$. The above results indicated that miR-495 interference plasmids were successfully constructed.

\section{Discussion}

OSCC represents the 6th most common cancers globally with reported incidence beyond 450,000 cases per annum and with 5-year survival rates of less than 50\% $[25,26]$. CSCs have drawn increasing attention due to their involvement in the progression of OSCC, and diverse miRNAs have been shown to regulate the cellular functions of CSCs from different types of cancers, such 


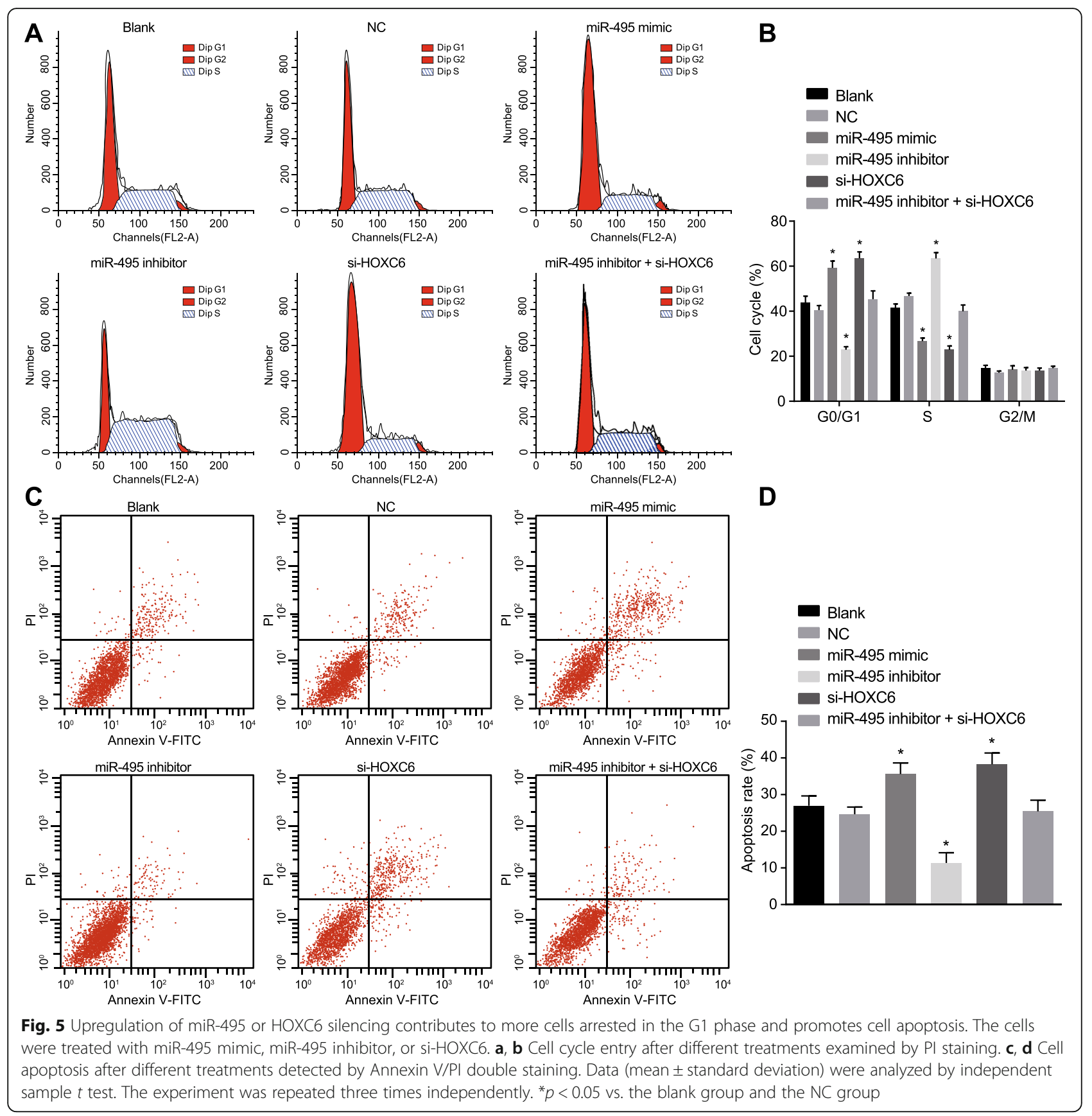

as prostate CSCs, as well as head and neck CSCs $[5,27]$. In this study, we examined the potential effects of miR495 on CSC proliferation, migration, and invasion in OSCC. Our findings confirmed that miR-495 had the capability to inhibit the TGF- $\beta$ signaling pathway by targeting HOXC6, thereby impairing CSC proliferation, migration, and invasion while inducing cell apoptosis in OSCC.

We initially found that the overexpressed miR-495 markedly inhibited the TGF- $\beta$ signaling pathway activation, manifested by reduced expression of the pathwayrelated genes (TGF- $\beta$, TGF $\beta$ RI, TGF $\beta$ RII, Smad2, and
Smad4), by suppressing HOXC6 in CSCs in OSCC. In line with our findings, miR-495 has been found to be downregulated in OSCC tissues and cell lines [28]. In the present study, the in silico analysis indicated that miR-495 could target HOXC6 and then repress its expression in vitro. Upregulation of miR-495 can reduce high glucose-induced inflammatory, cell differentiation, and extracellular matrix accumulation of human cardiac fibroblasts by blocking the nuclear factor $-\mathrm{kB}(\mathrm{NF}-\mathrm{\kappa B})$ and TGF- $\beta 1 /$ Smad signaling pathways [29]. The blockade of HOXC6-dependent TGF- $\beta$ signaling pathway is 


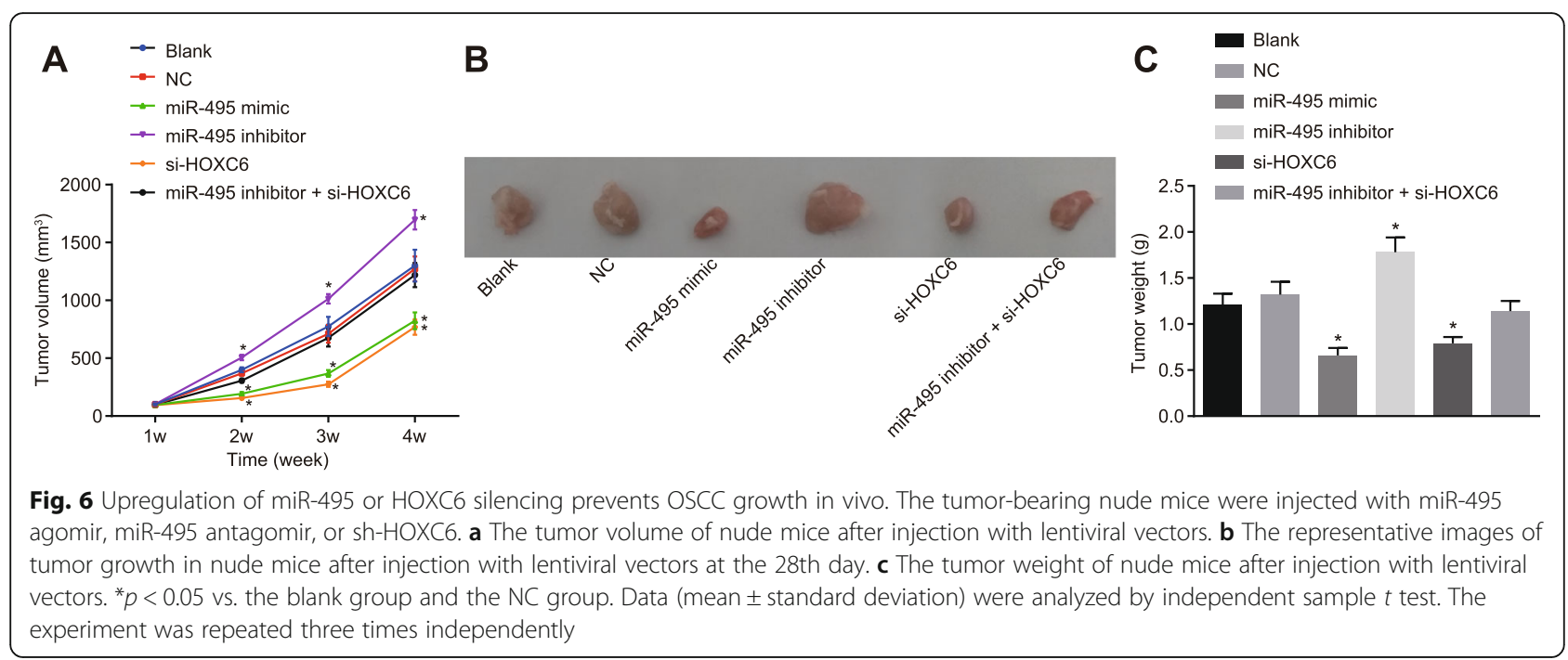

responsible for the suppressed EMT and lymph node metastasis in laryngeal cancer [30]. HOXC6 was found to be abundantly expressed in oral cancer FaDu-PTX cells compared to normal cells [31]. Moreover, the activated TGF- $\beta /$ Smad signaling pathway is capable to promote the proliferation and suppress the apoptosis of OSCC cells [32]. The above findings serve to illustrate an inverse correlation between miR-495 and HOXC6 and TGF- $\beta$ in OSCC.

Furthermore, forced miR-495 expression or HOXC6 silencing impeded EMT in OSCC cells, evidenced by obviously reduced levels of N-cadherin and Vimentin yet significantly elevated E-cadherin level in the miR-495 mimic and siHOXC6 groups. EMT is characterized by the loss of epithelial features, and the gain of mesenchymal features has been proposed to offer a connection between cancer metastasis and stem cell properties [1]. The importance of the EMT process in CSC formation has been stressed recently, and miRNAs have also been reported to be involved in controlling CSC functions and regulating cancer progression by affecting the EMT process [33]. Consistently, amplified miR495 expression has been shown to significantly inhibit EMT-

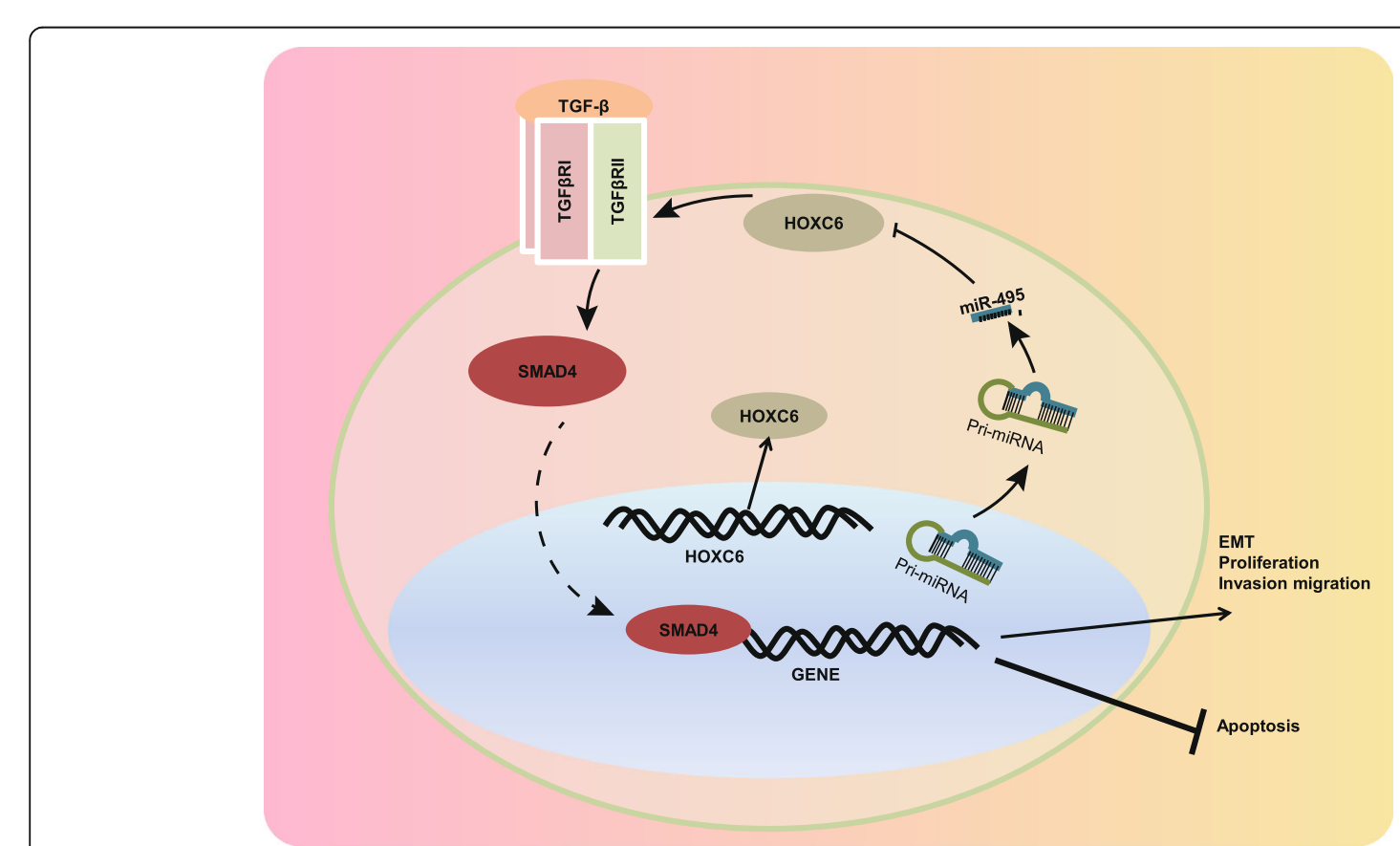

Fig. 7 The mechanism graph of the regulatory network of miR-495/HOXC6/TGF- $\beta$ in the progression of OSCC. miR-495 targets HOXC6 and subsequently represses its expression, leading to the blocked TGF- $\beta$ signaling pathway, ultimately impeding EMT, proliferation, invasion, and migration of CSCs while promoting cell apoptosis 
related proteins in OSCC cells in vitro [28]. A previous report showed that HOXB9 may promote OSCC EMT by inducing the activation of the TGF- $\beta 1 / \mathrm{Smad} 2 / \mathrm{Slug}$ signaling pathway [26]. TGF- $\beta$ is a key inducer of EMT [34]. The costimulation of TGF $\beta 1$ and EGF has been revealed to trigger the phenotype transition in OSCC cells, which meets the requirements of EMT accompanied by upregulated Vimentin and downregulated E-cadherin at the protein level [35]. A finding similar to ours was that HOXC6 gene silencing delays EMT (reflected by decreased N-cadherin and Vimentin expression and increased E-cadherin expression) through the inhibition of the activation of TGF- $\beta /$ Smad signaling pathway in cervical carcinoma cells [14].

Emerging evidence demonstrates that miRNAs play an important role in the regulation of cancer cell growth, invasion, and metastasis by inhibiting the expression of their targets [36]. In the current study, overexpression of miR-495 inhibited the proliferation, migration, and invasion while stimulating the apoptosis of CSCs in OSCC by downregulating its target HOXC6 gene. Similarly, the overexpression of miR495 inhibits OSCC cell proliferation and invasion through its suppression in Notch1 target gene in vitro [10]. One previous study suggested that $14 \mathrm{q} 32.31$ miRNAs, including miR-495, play important roles in the inhibition of cell proliferation, invasion, and migration in metastatic prostate cancer, where miR-495 could decrease cells in proliferative $S$ phase while increase cells in the G1 phase [37]. Another study suggested that the deregulation of HOX genes contributes to cancer development and progression [38]. A previous report revealed that miR-147-mediated HOXC6 silencing can inhibit cell proliferation and migration in hepatocellular carcinoma in vitro [39]. The overexpression of HOXC5 has also found to be associated with oral carcinogenesis and can significantly boost the development of OSCC [40]. Furthermore, siRNA against HOXC6 significantly reduces the growth of xenograft tumors in mice with oral cancer [31] and upregulation of miR-495 can inhibit the growth of OSCC xenografts in vivo [28], which were in agreement with our in vivo experimental results.

\section{Conclusion}

Our study demonstrates that miR-495 has the potential to retard CSC cell proliferation, invasion, and migration along with EMT while promoting cell apoptosis by perturbing the HOXC6-dependent TGF- $\beta$ signaling pathway activation (Fig. 7). These findings highlighted the potential of miR-495 as a new therapeutic intervention in the treatment of OSCC. Due to the limited sample size, the exact mechanism of miR-495 is not fully elucidated, and therefore, further large-scale studies are required to illustrate the underlying mechanism.

\section{Supplementary information}

Supplementary information accompanies this paper at https://doi.org/10. 1186/s13287-020-1576-3.

Additional file 1: Figure S1. Successful isolation of CSCs. A, The morphological observation of the purified OSCC cells $(\times 200)$. B and C, Immunohistochemistry analysis of Vimentin and keratin in OSCC cells ( $X$ 200)

Additional file 2: Figure S2. Sorting and identification of CSCs. A, The CD133 and CD44 positive cells sorted from OSCC cells detected by flow cytometry. B, The cell spheres enriched in OSCC cells detected by suspension sphere-forming test $(\times 200)$. C and D, The protein expression of CD133 and CD44 in OSCC cell spheres examined by western blot analysis. ${ }^{*} p<0.05$ vs. the OSCC cells. Data (mean \pm standard deviation) were analyzed by independent sample $t$-test. The experiment was repeated 3 times independently.

Additional file 3: Figure S3. Transfection efficiency of miR-495 overexpression or knockdown. ${ }^{*} p<0.05$ vs. the NC-mimic group. \# $p<0.05$ vs. the NC-inhibitor group. Data (mean \pm standard deviation) were analyzed by independent sample $t$-test. The experiment was repeated 3 times independently.

\section{Abbreviations}

3'-UTR: 3'-Untranslated region; ANOVA: Analysis of variance;

CDNA: Complementary DNA; CSCs: Cancer stem cells; DEGs: Differentially expressed genes; DMEM: Dulbecco's modified Eagle's medium;

DMSO: Dimethyl sulfoxide; EGF: Epithelial growth factor; EMT: Epithelialmesenchymal transition; FBS: Fetal bovine serum; FITC: Fluorescein isothiocyanate; GAPDH: Glyceraldehyde-3-phosphate dehydrogenase; GEO: Gene Expression Omnibus; HOXC6: Homeobox C6; IMDM: Iscove's modified Dulbecco's medium; LSD: Least significant difference; MEM: Minimum essential medium; NC: Negative control; NF-kB: Nuclear factor-kB; OD: Optical density; OSCC: Oral squamous cell carcinoma; PBS: Phosphate buffer saline; PMSF: Phenylmethanesulfonyl fluoride; PVDF: Polyvinylidene fluoride; RIPA: Radio-immunoprecipitation assay; RTqPCR: Reverse transcription quantitative polymerase chain reaction; SDAPAGE: Sodium dodecyl sulfate polyacrylamide gel electrophoresis; sh: Short hairpin RNA; si: Small interfering RNA; SP: Streptavidin peroxidase;

SPF: Specific pathogen-free; TBST: Tris-buffered saline with Tween-20; TGF$\beta$ : Transforming growth factor-beta; wt: Wild type

\section{Acknowledgements}

We would like to give our sincere appreciation to the reviewers for their helpful comments on this article.

\section{Authors' contributions}

$\mathrm{XY}$ and $\mathrm{ZZ}$ designed the study. WC, XW, and $\mathrm{HZ}$ collated the data, carried out data analyses, and produced the initial draft of the manuscript. WL revised the figures and tables. $\mathrm{XY}$ and $\mathrm{HZ}$ contributed to drafting the manuscript. All authors have read and approved the final submitted manuscript.

\section{Funding}

None.

\section{Availability of data and materials}

The datasets supporting the conclusions of this article are included within the article.

\section{Ethics approval and consent to participate}

The study was conducted under the approval of the Institutional Review Board of Jiangxi Cancer Hospital. Informed written consent was obtained from each participant prior to the study. Animal experiments were conducted under the approval of the Animal Ethics Committee of Jiangxi Cancer Hospital.

Consent for publication Not applicable. 


\section{Competing interests}

The authors declare that they have no competing interests.

\section{Author details}

'Department of Head and Neck Surgery, Jiangxi Cancer Hospital, No. 519, Beijing East Road, Nanchang 330029, Jiangxi Province, People's Republic of China. ${ }^{2}$ Department of Intensive Care Medicine, Jiangxi Cancer Hospital, Nanchang 330029, People's Republic of China. ${ }^{3}$ Department of Plastic Surgery, Jiangxi Cancer Hospital, Nanchang 330029, People's Republic of China. ${ }^{4}$ Department of Liver Oncology, Jiangxi Cancer Hospital, Nanchang 330029, People's Republic of China.

Received: 29 July 2019 Revised: 3 January 2020

Accepted: 29 January 2020 Published online: 14 March 2020

\section{References}

1. Ishida T, et al. Notch signaling induces EMT in OSCC cell lines in a hypoxic environment. Oncol Lett. 2013;6(5):1201-6.

2. Lohavanichbutr $P$, et al. Gene expression in uninvolved oral mucosa of OSCC patients facilitates identification of markers predictive of OSCC outcomes. PLoS One. 2012;7(9):e46575.

3. Costea DE, et al. Cancer stem cells - new and potentially important targets for the therapy of oral squamous cell carcinoma. Oral Dis. 2006;12(5):443-54.

4. Baillie $\mathrm{R}$, Tan ST, Itinteang T. Cancer stem cells in oral cavity squamous cell carcinoma: a review. Front Oncol. 2017;7:112.

5. Yu CC, et al. Targeting CD133 in the enhancement of chemosensitivity in oral squamous cell carcinoma-derived side population cancer stem cells. Head Neck. 2016;38(Suppl 1):E231-8.

6. Iorio MV, Croce CM. microRNA involvement in human cancer. Carcinogenesis. 2012;33(6):1126-33.

7. Lin S, Gregory RI. MicroRNA biogenesis pathways in cancer. Nat Rev Cancer. 2015;15(6):321-33.

8. Osaki M, Okada F, Ochiya T. miRNA therapy targeting cancer stem cells: a new paradigm for cancer treatment and prevention of tumor recurrence. Ther Deliv. 2015:6(3):323-37.

9. Ajemian I. Seminar on Canadian Medical Education in palliative care--a report. 7th International Congress on care of the terminally ill, Montreal, October 20, 1988. J Palliat Care. 1989;5(1):50-1.

10. Lv L, et al. MicroRNA495 targets Notch1 to prohibit cell proliferation and invasion in oral squamous cell carcinoma. Mol Med Rep. 2019;19(1):693-702.

11. Bitu CC, et al. HOXA1 is overexpressed in oral squamous cell carcinomas and its expression is correlated with poor prognosis. BMC Cancer. 2012;12: 146.

12. Du YB, et al. The survival predictive significance of HOXC6 and HOXC8 in esophageal squamous cell carcinoma. J Surg Res. 2014;188(2):442-50.

13. Hassan NM, et al. Aberrant expression of HOX genes in oral dysplasia and squamous cell carcinoma tissues. Oncol Res. 2006;16(5):217-24.

14. Zhang F, et al. HOXC6 gene silencing inhibits epithelial-mesenchymal transition and cell viability through the TGF-beta/smad signaling pathway in cervical carcinoma cells. Cancer Cell Int. 2018:18:204.

15. Javle $\mathrm{M}$, et al. Biomarkers of TGF-beta signaling pathway and prognosis of pancreatic cancer. PLoS One. 2014;9(1):e85942

16. Lu Z, et al. Tumor cell-derived TGF-beta at tumor center independently predicts recurrence and poor survival in oral squamous cell carcinoma. $J$ Oral Pathol Med. 2019;48(8):696-704.

17. Ayuk SM, Abrahamse $\mathrm{H}$, Houreld NN. The role of photobiomodulation on gene expression of cell adhesion molecules in diabetic wounded fibroblasts in vitro. J Photochem Photobiol B. 2016;161:368-74.

18. Wu F, et al. Paradoxical roles of TGF-beta signaling in suppressing and promoting squamous cell carcinoma. Acta Biochim Biophys Sin Shanghai. 2018;50(1):98-105

19. Jiang LL, Zhao YJ, Li Y. Expressions and clinical significance of TGF-betaRll and NF-kappaB in oral squamous cell carcinoma. Shanghai Kou Qiang Yi Xue. 2016;25(6):729-33.

20. Rivalland G, Scott AM, John T. Standard of care in immunotherapy trials: challenges and considerations. Hum Vaccin Immunother. 2017; 13(9):2164-78

21. Qiao B, et al. MicroRNA-27a-3p modulates the Wnt/beta-catenin signaling pathway to promote epithelial-mesenchymal transition in oral squamous carcinoma stem cells by targeting SFRP1. Sci Rep. 2017;7:44688.
22. Zeng G, et al. MicroRNA-27a-3p regulates epithelial to mesenchymal transition via targeting YAP1 in oral squamous cell carcinoma cells. Oncol Rep. 2016;36(3):1475-82.

23. Liu $B$, et al. MicroRNA-27b inhibits cell proliferation in oral squamous cell carcinoma by targeting FZD7 and Wnt signaling pathway. Arch Oral Biol. 2017:83:92-6.

24. Fukumoto I, et al. The tumor-suppressive microRNA-23b/27b cluster regulates the MET oncogene in oral squamous cell carcinoma. Int J Oncol. 2016:49(3):1119-29.

25. Ohta M, et al. Positive and negative regulation of podoplanin expression by TGF-beta and histone deacetylase inhibitors in oral and pharyngeal squamous cell carcinoma cell lines. Oral Oncol. 2013;49(1):20-6.

26. Xue $M$, et al. HoxB9 promotes the migration and invasion via TGF-beta1/ Smad2/Slug signaling pathway in oral squamous cell carcinoma. Am Transl Res. 2017:9(3):1151-61.

27. Chakraborty C, Chin KY, Das S. miRNA-regulated cancer stem cells: understanding the property and the role of miRNA in carcinogenesis. Tumour Biol. 2016;37(10):13039-48

28. Wang $Y$, et al. MiR-495/IGF-1/AKT signaling as a novel axis is involved in the epithelial-to-mesenchymal transition of oral squamous cell carcinoma. J Oral Maxillofac Surg. 2019;77(5):1009-21.

29. Wang $X_{\text {, et }}$ al. MicroRNA-495 inhibits the high glucose-induced inflammation, differentiation and extracellular matrix accumulation of cardiac fibroblasts through downregulation of NOD1. Cell Mol Biol Lett. 2018;23:23.

30. Chen $\mathrm{L}$, et al. Upregulation of microRNA-141 suppresses epithelialmesenchymal transition and lymph node metastasis in laryngeal cancer through HOXC6-dependent TGF-beta signaling pathway. Cell Signal. 2020; 66:109444.

31. Kim SA, et al. HOXC6 regulates the antitumor effects of pheophorbide abased photodynamic therapy in multidrug-resistant oral cancer cells. Int $J$ Oncol. 2016;49(6):2421-30.

32. Liu L, et al. Effects of IncRNA ANRIL on proliferation and apoptosis of oral squamous cell carcinoma cells by regulating TGF-beta/Smad pathway. Eur Rev Med Pharmacol Sci. 2019;23(14):6194-201.

33. Hao J, et al. MicroRNA control of epithelial-mesenchymal transition in cancer stem cells. Int J Cancer. 2014;135(5):1019-27.

34. Zaravinos A. The regulatory role of microRNAs in EMT and cancer. J Oncol. 2015:2015:865816.

35. Richter $\mathrm{P}$, et al. EGF/TGFbeta1 co-stimulation of oral squamous cell carcinoma cells causes an epithelial-mesenchymal transition cell phenotype expressing laminin 332. J Oral Pathol Med. 2011;40(1):46-54.

36. Chan SH, Wang LH. Regulation of cancer metastasis by microRNAs. J Biomed Sci. 2015:22:9.

37. Formosa A, et al. MicroRNAs, miR-154, miR-299-5p, miR-376a, miR-376c, miR 377, miR-381, miR-487b, miR-485-3p, miR-495 and miR-654-3p, mapped to the $14 q 32.31$ locus, regulate proliferation, apoptosis, migration and invasion in metastatic prostate cancer cells. Oncogene. 2014;33(44):5173-82.

38. Aquino $\mathrm{G}$, et al. Deregulation of paralogous $13 \mathrm{HOX}$ genes in oral squamous cell carcinoma. Am J Cancer Res. 2015:5(10):3042-55.

39. Sui CJ, et al. MicroRNA-147 suppresses human hepatocellular carcinoma proliferation migration and chemosensitivity by inhibiting HOXC6. Am J Cancer Res. 2016:6(12):2787-98.

40. Moon SM, et al. Homeobox C5 expression is associated with the progression of 4-nitroquinoline 1-oxide-induced rat tongue carcinogenesis. J Oral Pathol Med. 2012;41(6):470-6.

\section{Publisher's Note}

Springer Nature remains neutral with regard to jurisdictional claims in published maps and institutional affiliations. 